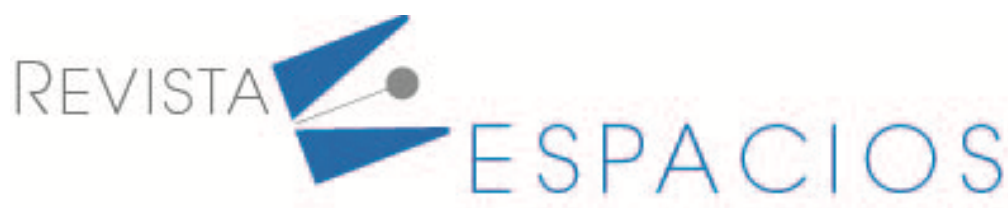

Vol. 41 (47) $2020 \cdot$ Art. 4

\title{
Manejo oportuno de escombros en Villavicencio Meta, Colombia
}

\section{Timely management of rubble in Villavicencio Meta, Colombia}

\author{
AGUDELO VARELA, Mateo ${ }^{1}$ \\ RODRÍGUEZ MIRANDA, Juan Pablo ${ }^{2}$ \\ MESA FERNÁNDEZ, Duván Javier ${ }^{3}$
}

\begin{abstract}
RESUMEN
Uno de los mayores consumidores de recursos naturales a nivel mundial es el sector de la construcción, utiliza grandes volúmenes de áridos en la elaboración de concretos y genera cantidades considerables de residuos sólidos, denominados escombros, producto de las demoliciones, construcciones y remodelaciones de obras civiles. El manejo de los residuos de construcción y demolición (RCD) constituye uno de los principales retos a resolver por las empresas constructoras y entidades gubernamentales. En la ciudad de Villavicencio el manejo inadecuado que han recibido los escombros en las últimas décadas ha generado impactos negativos como disposición incontrolada de material particulado, contaminación visual, alteraciones del paisaje, uso impropio de medios de transporte y proliferación de malas prácticas de reciclaje. Se estima que para el año 2013 Villavicencio generó un aproximado de $107.180 \mathrm{~m}^{3}$ de residuos de construcción lo que equivale a $0,95 \mathrm{~kg} / \mathrm{hab} / \mathrm{día}$, los cuales al no contar con escombrera legal fueron dispuestos inadecuadamente en diversos puntos de la ciudad. Debido a esto urge en Villavicencio la aplicación del manejo oportuno de los RCD, que consiste en un conjunto de actividades que se fundamentan en disminuir, reusar, reciclar, disponer y eliminar de forma controlada los RCD, lo que generará beneficios como la disminución de residuos, la reducción del impacto ambiental, ahorro de energía, generación de empleo y mejora la imagen corporativa de las empresas frente a la comunidad.
\end{abstract}

Palabras clave: CDW, reutilización, gestión.

\begin{abstract}
Summary
One of the largest consumers of natural resources worldwide is the construction sector, it uses large volumes of aggregates in the production of concrete and generates considerable amounts of solid waste, called rubble, as a result of demolition, construction and remodeling of civil works . The management of construction and demolition waste (RCD) constitutes one of the main challenges to be solved by construction companies and government entities. In the city of Villavicencio the inadequate management that the rubble has received in the last decades has generated negative impacts such as uncontrolled disposal of particulate matter, visual contamination, landscape alterations, improper use of transportation means and proliferation of
\end{abstract}

\footnotetext{
${ }^{1}$ Ingeniero Civil. M.Sc.Gestión Ambiental Sostenible. Profesor asociado, Facultad de Ingenierías, Universidad Cooperativa de Colombia, Villavicencio, Meta, Colombia. Correo electrónico: mateo.agudelov@campusucc.edu.co;

2 Profesor Titular. Universidad Distrital Francisco José de Caldas. Director del grupo de investigación AQUAFORMAT Correo electrónico: jprodriguezm@udistrital.edu.co

${ }^{3}$ Ingeniero Ambiental. M.Sc. en Ciencias Ambientales cPh.D. Tecnología y Administración del Agua TAYGA Universidad de Murcia España. Dirección postal: Cra. No. 51-11 Bogotá D.C.Colombia duvanmesa@usantotomas.edu.co
} 
bad recycling practices. It is estimated that for the year 2013 Villavicencio generated an approximate of $107,180 \mathrm{~m} 3$ of construction waste, which is equivalent to $0.95 \mathrm{~kg} /$ inhab / day, which, since they did not have a legal waste dump, were improperly disposed of in various parts of the city. Due to this, Villavicencio urges the application of the timely management of the RCD, which consists of a set of activities that are based on reducing, reusing, recycling, disposing and eliminating the RCD in a controlled way, which will generate benefits such as the reduction of waste, the reduction of environmental impact, energy saving, job creation and improves the corporate image of companies vis-à-vis the community.

Key Words: CDW, reuse, management.

\section{Introducción}

El crecimiento constante de la población demanda cada vez más recursos naturales y energía para abastecer los procesos de producción de bienes y servicios de diferentes sectores económicos, siendo uno de los sectores más grandes el de la construcción (Bernardo et al. 2016). Relevancia que debe orientar los diferentes procesos del sector de la construcción en concordancia con los objetivos de desarrollo sostenible establecidos por las Naciones Unidas.

No obstante, un gran número de actividades de construcción, renovación y demolición de obras civiles tienen lugar en todo el mundo en forma continua. Estas actividades dan como resultado enormes volúmenes de residuos de construcción y demolición (RCD) que tienen que ser eliminados y manejados, con graves implicaciones financieras y ambientales (Bernardo et al. 2016). Generando una problemática de gran importancia para la sociedad moderna, ya que representan aproximadamente el $35 \%$ de todos los residuos industriales producidos en todo el mundo (Alencar et al. 2011), alcanzando en algunas ciudades hasta un $40 \%$ del flujo total de residuos (Hoornweg et al. 2012), sin embargo, estudios recientes afirman que los RCD aportan entre el 25 y 30\% del total de los residuos sólidos generados a nivel mundial anualmente (Rodríguez et al. 2015).

Concomitantemente a lo anterior, en las últimas décadas el crecimiento económico ha generado grandes avances sociales lo que ha permitido que los indicadores económicos hayan sido durante años sistemáticamente positivos, mientras que los indicadores ambientales resultan cada vez más negativos (Vásquez et al. 2015). Convirtiéndose el sector de la construcción en uno los principales contribuyentes a la actual problemática ambiental generada por el consumo excesivo de los recursos naturales (De Brito et al. 2010) y los grandes volúmenes de residuos generados. Acentuándose significativamente los problemas relacionados con la generación y manejo de residuos sólidos sino se cuenta con buenas prácticas de ejecución en las obras civiles (Hoffmann et al. 2012).

Dichos residuos sólidos son denominados escombro o RCD, y su definición según el Concejo Municipal de Villavicencio en el acuerdo 047 del 4 de mayo de 2009 es: "Todo tipo de residuo sólido, resultante de demoliciones, reparación de inmuebles o construcciones de obras civiles; es decir, los sobrantes de cualquier acción que se ejerza en las estructuras urbanas y rurales".

Para dar una idea de la importancia de la generación de los RCD a nivel nacional se toma como referente la ciudad de Bogotá y la estimación que alude Silva et. al (2015), de quince millones $\mathrm{m}^{3}$ /año o de dos toneladas per cápita. Esta cifra representa una magnitud de producción alarmante al ser comparada con el promedio europeo antes de la crisis del 2010, sólo países como Dinamarca, Finlandia, Alemania, Irlanda y Luxemburgo, presentaron cantidades superiores a 2t/per cápita/año. Más desalentador resulta el panorama al revisar las cifras de reutilización de estos mismos países, los cuales superan el 50\% de aprovechamiento de los RCD en comparación con el 5 al 10\% que reutiliza o recicla la ciudad de Bogotá y resto de Colombia (Castaño et al. 2013).

En el ejercicio profesional de la construcción se producen residuos sólidos en las distintas etapas de los proyectos, tales como: volúmenes considerables de tierra durante la excavación, concretos y pedazos de hierro durante la 
estructura, mampuestos y mortero durante la mampostería, retal de cerámica, vidrios y pintura, entre otros, durante los acabados y según Serrano et al. (2009) se considera que la cantidad y composición de estos es variada, es preciso estimar estos dos últimos factores como punto de partida para la gestión que se aplique sobre los RCD. Conviene distinguir que hoy en día el manejo de los RCD apunta a la reutilización de los escombros de concreto, sin embargo estos no reciben la importancia que merecen y se restringe su uso principalmente a material de relleno, material de sub-base, material para los niveles básicos en la construcción de carreteras y como agregado en concreto nuevo para elementos no estructurales, de acuerdo con lo reportado por Sui \& Mueller (2012) y el uso de agregados reciclados para elaboración de concreto se están estudiando intensamente desde hace unos 30 años (Hoffmann et al., 2012).

Adicional a lo anterior, el objetivo de la gestión de los RCD es hacer uso eficiente de los recursos sobre la base de las 3R según lo postulado por Yeheyis (2013), en el que la reducción o prevención es la primera opción de gestión, seguida de la reutilización, reciclado, valorización y la disposición en escombreras, por último, direccionando así a la sociedad a un escenario donde los residuos se conviertan en materias primas y se reincorporen a la cadena productiva.

Es así como desde la gestión de los RCD y de la recolección directa e indirecta de información este documento plasma el escenario actual de la ciudad de Villavicencio desde una perspectiva económica, social y ambiental frente al manejo de los escombros, que permita identificar tanto la problemática generada por los residuos así como la aceptación de la población de productos generados a partir del reciclaje de los RCD con el ánimo de proponer alternativas de manejo adecuado de los escombros para la ciudad.

\section{Materiales y métodos}

El tipo de investigación aplicada a este desarrollo del manejo de los escombros puede ser establecido de acuerdo con el planteamiento prospectivo de Vergel (2010), dado que se registra información según va ocurriendo el fenómeno, en este caso, obtener los datos de los escombros en la ciudad de Villavicencio; sin embargo, según el análisis y alcance de los resultados la investigación también puede ser cuasi experimental debido a que existe una relación causal entre las cantidades, tipo y composición de los residuos de escombros, que en condiciones de control riguroso de los factores pueden afectar el resultado del análisis. Además, conforme a la información recolectada se puede proponer alternativas de manejo adecuado de los escombros para la preservación y conservación de cuencas hídricas en el departamento, por ello según Hurtado (2000), la investigación también se denomina de pronóstico. De esta forma se puede señalar tres grandes fases que contemplan la total ejecución del proceso de investigación:

Fase organizativa: En esta etapa inicial a partir de la observación directa se logra identificar la problemática que da lugar a este estudio delimitándolo espacial y temporalmente.

Fase aplicada: En esta fase se contempla la descripción de la metodología a seguir, la población y muestra con la que se trabaja, el diseño y verificación de las herramientas de recolección de información mediante observación de campo, dos encuestas semi estructuradas y medición del tipo y cantidad de escombros generados. Finaliza con la aplicación de las herramientas depuradas sobre la muestra seleccionada junto con el análisis de los datos.

Fase de integración: Esta es la fase final del proyecto, en donde se incorporan los hallazgos obtenidos, las conclusiones y algunas recomendaciones para lograr avanzar en la solución al problema visualizado en un comienzo.

Población y selección de la muestra: La población comprende los residuos de construcción generados durante el periodo de ejecución de una obra civil seleccionada con licencia de construcción en la ciudad de Villavicencio durante los meses de diciembre 2013 a junio 2014.Se identificaron las obras más representativas en los últimos 
años con la información recopilada por el DANE (2014a) del área aprobada en metros cuadrados según destino entre enero 2007 a diciembre 2013 para la ciudad. Se identificaron los usos más representativos que son residenciales y comerciales con un porcentaje del 79,76 y 6,99\%. A partir de este punto y conforme al método en investigación cuantitativa - triangulación, se tomaron tres obras de cada uno de los dos usos más representativos para la toma de las muestras representativas en cada una de ellas. Para un total de seis obras (3 residenciales y 3 comerciales).

Para las obras destinadas a uso residencial y comercial seleccionadas se evaluaron los RCD generados, se clasificaron y se estimó el factor de generación en $\mathrm{m}^{3}$ de residuos por $\mathrm{m}^{2}$ construido. El número de muestras en cada obra se realizó según Triola (2009). La estimación cuando se desconoce la población puede calcularse a partir de la siguiente ecuación:

$$
\begin{aligned}
& \mathrm{n}=\frac{\left[\frac{Z \propto / 2}{2}\right]^{2} \cdot 0,25}{E^{2}} \\
& \mathrm{n}=\text { Tamaño de la muestra } \\
& \mathrm{Z}_{\propto / 2}=\text { Valor crítico } \\
& \mathrm{E}=\text { margen de error }
\end{aligned}
$$

Se empleó un margen de error del 8\%, y un valor crítico del 1,96 que representa un nivel de confianza del 95\% $(P<0,05)$ se obtuvo un tamaño de la muestra de 150 . El proceso para la toma de muestras conlleva cuatro pasos: toma de la muestra; peso total de muestra; clasificación y peso de cada componente. Concomitantemente a lo anterior las otras herramientas utilizadas para la recolección de la información son: observación en campo y aplicación de las encuestas.

Herramientas de recolección de información. Observación en campo: realizada a las seis obras seleccionadas para la cuantificación de los RCD generados y de otras 62 observaciones puntuales realizadas en diferentes obras de la ciudad. Conforme a los resultados obtenidos al aplicar las herramientas de recolección de información se construirá una tabla que esboce la problemática por cada una de las fases de los RCD en tres aspectos que enmarcan los escombros; económico, social y ambiental.

\section{Resultados y discusión}

El tema del reciclaje de RCD ha sido objeto de estudios e investigaciones desde la década de 1970 y las principales razones de estos estudios son la creciente conciencia de la importancia del reciclaje de residuos de todas las fuentes en el contexto del desarrollo sostenible (Pacheco et al. 2013). Manteniendo ese contexto, cada uno de los instrumentos mencionados se aplicaron a las muestras seleccionadas y los resultados obtenidos fueron:

Herramienta 1 medición de escombros. Los resultados obtenidos de la generación de residuos de construcción RCD de obras para uso residencial y para uso comercial son de 0,137 a $0,151 \mathrm{~m}^{3} / \mathrm{m}^{2}$ y de 0,076 a $0,086 \mathrm{~m}^{3} / \mathrm{m}^{2}$ respectivamente. Así mismo de acuerdo al proceso de separación y pesaje de cada muestra se obtuvieron los porcentajes de composición de los RCD según el contenido de agregado grueso, agregado fino, mampostería, concreto, acero y otros. El componente de otros incluye; madera, plástico, yeso, PVC y residuos peligrosos. A partir de los índices determinados y de acuerdo a la información presentada por el DANE (2014a) del área licenciada para construir en Villavicencio se puede estimar la cantidad de escombros generados en la ciudad. Para ello se usó el índice mínimo y máximo obtenido de las obras destinadas a uso vivienda ya que estas representan alrededor del $80 \%$ del área licenciada en la ciudad en los últimos siete años. Así mismo se consideró una fuga de escombros debido a las obras sin licencia que se ejecutan en la ciudad, según lo expresa la Alcaldía municipal el porcentaje de ilegalidad ronda el $60 \%$, por lo tanto, para efectos de la estimación total de los RCD 
se toma el índice promedio de generación de escombros para uso vivienda, $0,144 \mathrm{~m}^{3} / \mathrm{m}^{2}$, se multiplica por los metros cuadrados de área licenciada y se aumenta la fuga correspondiente al $60 \%$. Los resultados obtenidos se presentan en la tabla 1. Adicionalmente tomando el total estimado de RCD y la población proyectada por el DANE (2014b) en Villavicencio para el año 2013 se obtiene un valor de RCD de 0,95Kg/hab./día.

Tabla 1

Estimado de escombros generados en Villavicencio

\begin{tabular}{|c|c|c|c|c|}
\hline Año & $\begin{array}{c}\mathrm{m}^{2} \text { licenciados para } \\
\text { construcción }\end{array}$ & $\begin{array}{c}\mathrm{RCDm}{ }^{3} \times 10^{3} \\
\text { Índice Máximo }\end{array}$ & $\begin{array}{c}\mathrm{RCDm} 3 \times 10^{3} \\
\text { Índice Mínimo }\end{array}$ & $\begin{array}{c}\text { Total RCDm } \\
\mathrm{x} 10^{3} \text { inc. Fugas }\end{array}$ \\
\hline 2001 & 75.002 & 11,33 & 10,28 & 17,28 \\
\hline 2002 & 217.064 & 32,78 & 29,74 & 50,01 \\
\hline 2003 & 170.082 & 25,68 & 23,30 & 39,19 \\
\hline 2004 & 170.742 & 25,78 & 23,39 & 39,34 \\
\hline 2005 & 257.979 & 38,95 & 35,34 & 59,44 \\
\hline 2006 & 164.221 & 24,80 & 22,50 & 37,84 \\
\hline 2007 & 205.404 & 31,02 & 28,14 & 47,33 \\
\hline 2008 & 184.213 & 27,82 & 25,24 & 42,44 \\
\hline 2009 & 310.818 & 46,93 & 42,58 & 71,61 \\
\hline 2010 & 282.292 & 42,63 & 38,67 & 65,04 \\
\hline 2011 & 456.695 & 68,96 & 62,57 & 105,22 \\
\hline 2012 & 385.760 & 58,25 & 52,85 & 88,88 \\
\hline 2013 & 465.207 & 70,25 & 63,73 & 107,18 \\
\hline
\end{tabular}

Figura 1

Estimado de escombros generados en Villavicencio

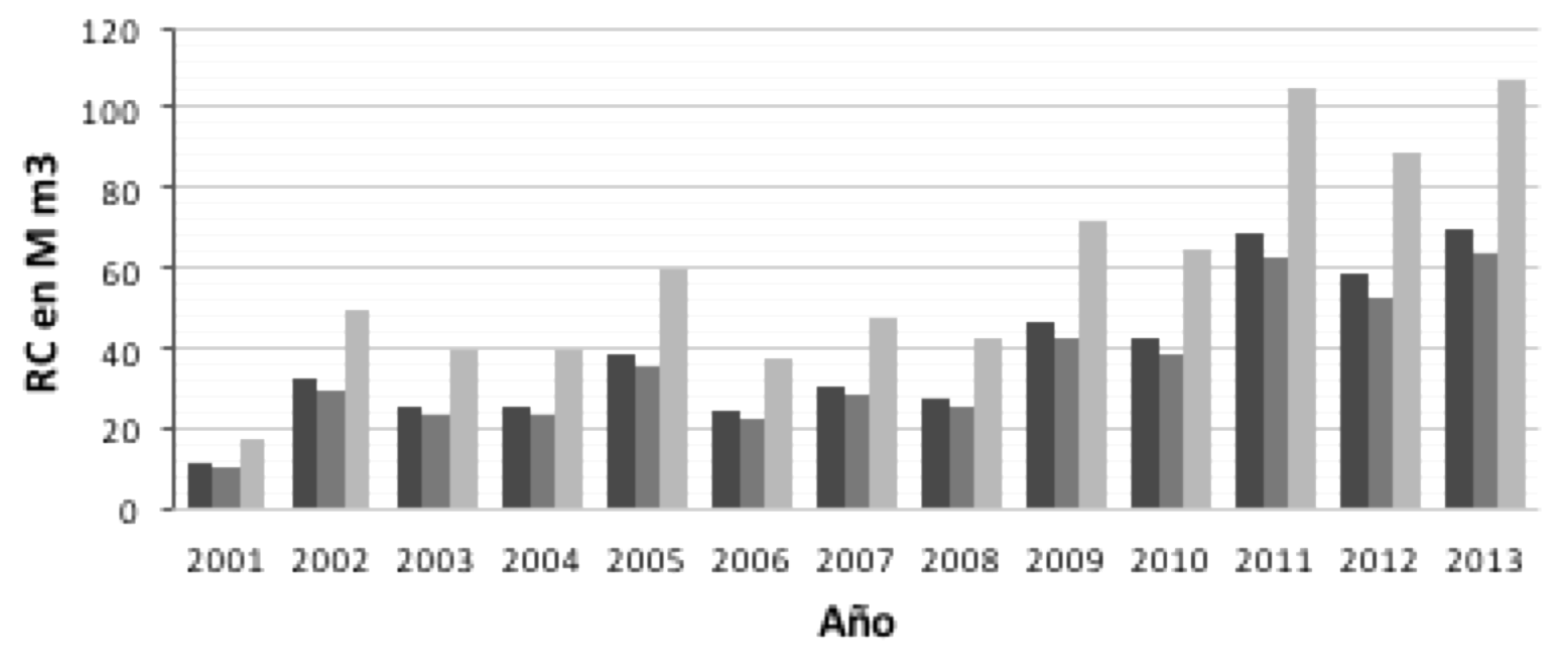

- RC m3 x103 Indice Mavimo $\quad \mathrm{RC}$ m3 x103 Indice Minimo - Total RC m3 x103 inc. Fugas

Herramienta 2 encuestas semiestructuradas. A partir de la información suministrada por los participantes a las encuestas semiestructuradas se obtuvieron los resultados que se observan en la figura 2 donde se recalca el desconocimiento a la legislación ambiental para la ciudad con un 95\% tanto de los constructores como de la población en general, así mismo el 78\% de los constructores encuestados desconoce el lugar de disposición final de los escombros generados. Estos dos resultados demuestran la falta de interés que presentan los constructores frente al manejo adecuado de los escombros generados. Por otro lado, se encontró que el $2 \%$ de la población en 
general considera el RCD como un material con potencial reúso frente a un $24 \%$ de los constructores. No obstante, el 73\% de la población en general está de acuerdo con el reúso de los RCD en obras públicas y un 77\% de los constructores usaría materiales reciclados si fueran más económicos que los de origen natural, evidenciando esto un gran potencial de reúso de los escombros en la ciudad debido a la aceptación social y económica de estos.

Herramienta 3 observación en campo. De los resultados obtenidos de la observación en campo se resaltan:

- El proceso constructivo ejecutado obedece principalmente a prácticas artesanales.

- Escasa presencia de personal profesional en la ejecución de obras civiles destinadas a uso residencial.

- Los profesionales a cargo de la ejecución de las obras civiles desconocen la normatividad ambiental que enmarca los RCD y no aplican buenas prácticas de manejo de RCD en las obras.

- Las actividades encaminadas a la separación de los RCD en sitio son dirigidas principalmente a la madera.

- La reutilización de los RCD se da como relleno in situ principalmente o en un predio privado.

- El costo de transporte a usar para los RCD es uno de los factores diferenciadores.

- Desinterés por parte de los generadores en cuanto al manejo de los RCD puertas hacia afuera y su sitio de disposición final.

Así mismo la observación en campo se orientó hacia la identificación del comportamiento de los diferentes actores respecto al manejo de los escombros en la ciudad. Los actores identificados son las secretarias de planeación y medio ambiente de la alcaldía municipal, Cormacarena, Bioagrícola, Policía Nacional, curadurías urbanas, personería, generadores, transportadores y comunidad en general. El dialogo entre los actores que están involucrados en el manejo de los escombros en la ciudad de Villavicencio es descoordinado lo cual genera los vacíos que permiten a los generadores y transportadores realizar prácticas inadecuadas en el manejo de los $R C D$, en ocasiones a la vista de la comunidad, sin que esto acarree las sanciones estipuladas por la legislación vigente. Adicionalmente, se identificó el ciclo actual de los RCD en la ciudad de Villavicencio, el cual está compuesto por tres fases: generación, transporte y disposición final, enmarcadas por condiciones como: no contar con un sitio autorizado para la disposición final de los RCD, bajo interés por parte de la comunidad hacia el manejo adecuado de estos, uso inapropiado de medios de transporte y poco control por parte de las autoridades, lo que genera un escenario que propicia la inadecuada disposición de los RCD en rondas de ríos, espacio público, vías, separadores, predios públicos y privados.

Conforme a los resultados obtenidos para la ciudad de Villavicencio se estima un índice de generación de escombros en $0,144 \mathrm{~m}^{3} / \mathrm{m}^{2}$, mientras que en España se estima para los nuevos edificios un volumen de residuos de construcción, sin tierra, de $0,125 \mathrm{~m}^{3} / \mathrm{m}^{2}$ construido, y en demoliciones un volumen de 0,732 a $0,874 \mathrm{~m}^{3} / \mathrm{m}^{2}$ demolido. Sin embargo, se estima que por cada $1.000 \mathrm{~m}^{2}$ construidos se genera como residuo $15 \mathrm{~m}^{3}$ de estructura según lo establecido por el MMAE en (2001), $55 \mathrm{~m}^{3}$ de cerramientos y $50 \mathrm{~m}^{3}$ de acabados. Por otro lado, según lo estimado por D'APPOLONIA (1999) por cada $\mathrm{m}^{2}$ de construcción nueva se generan alrededor de $0,5 \mathrm{~m}^{3}$ de residuos. En la misma línea Muñoz (2013) estimó un índice de generación de residuos de $0,14 \mathrm{~m}^{3} / \mathrm{m}^{2}$ construido, mientras Fernández (2013) indica que por cada $\mathrm{m}^{2}$ construido, se genera $1,42 \mathrm{~m}^{3}$ de escombros incluyendo material de excavación.-En cuanto a la composición de los RCD existen datos de diferentes autores que muestran que la mayor fracción obedece a la suma del concreto y la mampostería superando el 50\% del total de los RCD.

La problemática en torno a los RCD se intensifica cuando se unen el gran volumen que se genera en los centros poblados, la variada composición de estos y una inadecuada gestión generándose escenarios como los observados en la ciudad de Villavicencio, donde los RCD tiene como sistema de gestión, el vertido controlado en 
contadas ocasiones, pero sobre todo incontrolado, los sitios autorizados están totalmente diseminados, con escasas alternativas de valorización, reciclaje o reutilización (Castaño et al. 2013).

De dicha problemática surge la necesidad de dar manejo adecuado a los RCD generados, lo que plantea la gestión como pilar principal del proceso. La premisa es romper la relación directa entre el crecimiento económico y el aumento de la generación de residuos, la reducción de los impactos ambientales asociados y fomentar el reciclaje y la sostenibilidad (Dahlbo et al. 2015). Se plantea como alternativa para la gestión de los RCD en la ciudad de Villavicencio un manejo coordinado de escombros que permitirá administrar los RCD en cada una de sus fases basado en los principios de disminución, reutilización, reciclaje y eliminación que permita la obtención de beneficios como disminución de RCD, reducción del impacto ambiental, ahorro de energía y mejora de imagen corporativa.

En la fuente. Procesos constructivos que generen menos residuos y/o permitan reciclaje más eficiente; separación de los RCD según el potencial de reutilización o reciclaje, uso de contenedores o áreas que impidan la mezcla con otros materiales y/o el ambiente; supervisiones calificadas de la ejecución de la obra y manejo de los RCD generados; aplicar la normativa ambiental nacional y local vigente para el manejo de los RCD, con énfasis en el decreto 089 del 26 de mayo de 2014, por medio del cual se regula la gestión integral de escombros en el municipio de Villavicencio. Adicionalmente, aplicar usos alternativos a los RCD como la producción de material granular fino o arena para concretos o morteros (Costafreda et al. 2013) y como agregado grueso para concreto (Hoffmann et al. 2012)

En el transporte. Renovar y especializar el parque automotor encargado del traslado de los RCD a centros de acopio o escombrera autorizada; financiar e incorporar a los transportadores informales al pie de fuerza laboral que transporte residuos especiales a centros de acopio temporal, aplicar y controlar la normativa ambiental nacional y local vigente para el manejo de los RCD, haciendo énfasis en el decreto 089 del 26 de mayo de 2014, por medio del cual se regula la gestión integral de escombros en el municipio de Villavicencio.

En el sitio de disposición final. Recepción y clasificación de los RCD en acopios diferentes para ser direccionados a plantas que se encarguen trituración y tamizado para posterior reúso. El sitio de disposición final deberá contar con: 1) una planta de procesamiento de las fracciones de concreto, agregados y mampostería convirtiéndose así no solo en una escombrera sino también en una planta de suministro de agregados provenientes del reciclaje de los escombros, 2) instalación de centros pilotos de acopio temporal de RCD y residuos especiales en diferentes puntos de la ciudad.

De acuerdo a las tres fases anteriores (fuente, transporte y disposición final) y con la información recolectada se construye la tabla 2 que presenta la problemática que generan los RCD en la ciudad de Villavicencio en los aspectos económico, social y ambiental. Lo cual permitirá diseñar un sistema encaminado a minimizar la generación de RCD y el impacto que estos generan en el entorno, así como estimular el reciclaje y reúso de estos residuos. Para el diseño de dicho sistema se requiere de una integración completa de los eslabones que componen la cadena de gestión. En estos términos, la divulgación de información, la educación, la aplicación de incentivos y nuevas tecnologías son esenciales para direccionar el comportamiento de los actores hacia un manejo oportuno de los RCD en la ciudad de Villavicencio apuntando a una economía circular como se plasma en la figura 3. 
Tabla 2

Problemática por fases de los RCD en Villavicencio

\begin{tabular}{|c|l|l|l|}
\hline \multirow{2}{*}{ Fase } & \multicolumn{3}{|c|}{ Problemática } \\
\cline { 2 - 4 } Generación & $\begin{array}{l}\text { Aspecto económico } \\
\text { Pérdida de valor en los RCD } \\
\text { con potencial de } \\
\text { reutilización y/o reciclaje }\end{array}$ & $\begin{array}{l}\text { Aso inadecuado de } \\
\text { espacio público para } \\
\text { acopio temporal de RCD }\end{array}$ & $\begin{array}{l}\text { Fuga de material a las redes } \\
\text { de alcantarillado }\end{array}$ \\
\hline Transporte & $\begin{array}{l}\text { Aumento de costo del } \\
\text { transporte por RCD con } \\
\text { potencial de reutilización }\end{array}$ & $\begin{array}{l}\text { Uso de vehículo de } \\
\text { tracción animal y otros } \\
\text { inadecuados }\end{array}$ & $\begin{array}{l}\text { Aumento de material } \\
\text { particulado, consumo } \\
\text { adicional de energía }\end{array}$ \\
\hline Disposición final & $\begin{array}{l}\text { Pérdida de valorización del } \\
\text { predio de disposición final y } \\
\text { aledaños. }\end{array}$ & $\begin{array}{l}\text { Proliferación de prácticas } \\
\text { inadecuadas de reciclaje } \\
\text { de los RCD }\end{array}$ & $\begin{array}{l}\text { Alteraciones del paisaje, } \\
\text { cambios en la estabilidad } \\
\text { del suelo y contaminación } \\
\text { de fuentes hídricas }\end{array}$ \\
\hline
\end{tabular}

Teniendo en cuenta que, las principales barreras para el reciclaje de los RCD son la alta disponibilidad y bajo costo de las materias primas vírgenes, que según Dahlbo et al. (2015) disminuyen la demanda de productos reciclados y el interés en el desarrollo de negocio de reciclaje, es preciso difundir los resultados de múltiples estudios que demuestran que el uso de los RCD como agregado es viable para la fabricación de concretos, tal y como lo expresa Eljaiek et al. (2011). Esto permitirá denotar la importancia del potencial de reciclaje que se estima en $100 \%$ de acuerdo con Chong \& Hermreck, (2010), siempre que no esté contaminado en concordancia con lo planteado por Natalini et al. (2000). Así mismo a los RCD puede dársele un uso amplio en las obras civiles que en atención a lo formulado por Wrap (2002) abarca desde los cimientos, la superestructura, elementos no estructurales, vías hasta obras hidráulicas, lo que ocasionará la disminución de costos y ventajas en aspectos ambientales (Silva et al. 2015). En concordancia a lo anterior la competitividad del reciclaje podría aumentarse con alzas en el precio de las materias primas primarias a través de impuestos.

Figura 2

Resultados encuesta a población y constructores

\section{Encuestas a población}

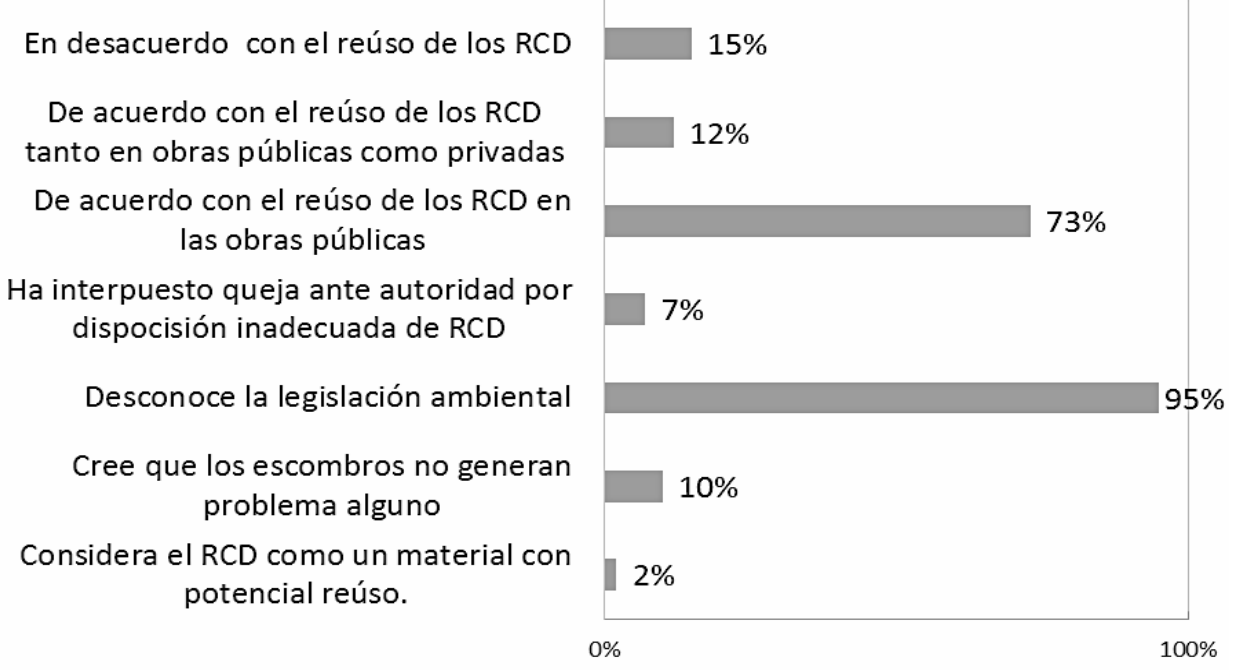

Muestra 145 hab. 


\section{Encuestas a Constructores}

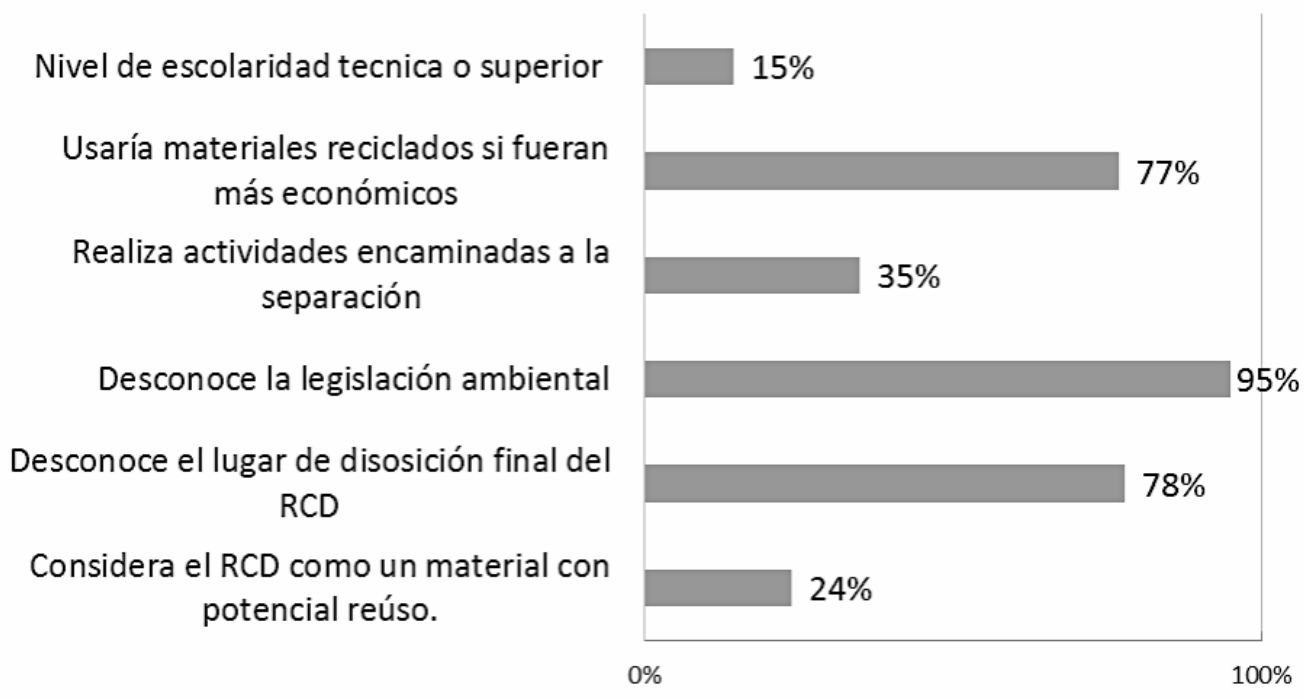

Muestra 68 obras

Figura 3

Manejo oportuno de escombros

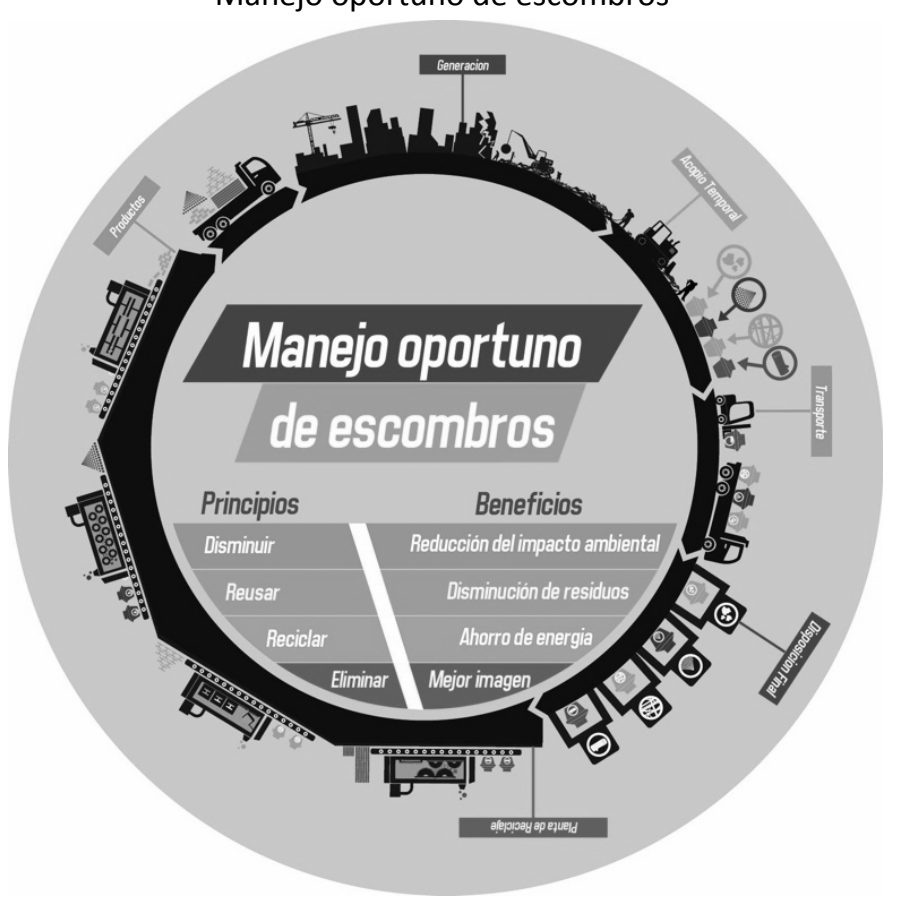

Casos exitosos en diferentes países del mundo demuestran que es fundamental que la población participe activamente en la gestión de los RCD, toda vez, que son ellos los generadores del residuo y consumidores de los productos generados. Siendo de esta manera necesario abordar los aspectos sociales, económicos y ambientales que inciden en los diferentes actores de la gestión de los RCD.

Desde el punto de vista económico, se requiere evaluar el proceso de separación en la fuente, transporte, reciclaje y venta de productos generados en el reciclaje. Desde lo social, estimar la generación de empleo formal, la formulación de campañas de educación y el cambio de medios de transporte. Desde lo ambiental, la estimación de las alteraciones al entorno. 
No obstante, la ciudad de Villavicencio ha padecido la indiferencia de sus dirigentes y habitantes en cuanto al manejo adecuado de los escombros se refiere. Los residuos provenientes de la demolición total o parcial de estructuras existentes y la ejecución de obra nueva son dispuestos en sitios inadecuados que no cumplen con la legislación ambiental vigente sobre el tema. La ciudad de Villavicencio generó en 2013 un aproximado de $107.180 \mathrm{~m}^{3}$ de RCD lo que permite inferir un índice de generación de 0,95kg/hab/día.

El comportamiento actual de los generadores de RCD en la ciudad de Villavicencio frente a la gestión de estos se ve en gran medida influenciada por la tardía aplicación de la normativa ambiental nacional por parte de las autoridades municipales, afianzando en la comunidad el concepto que es más económico contaminar que dar un manejo oportuno a los RCD. El manejo oportuno de los (RCD), son un conjunto de actividades de gran importancia e impacto ambiental, social y económico que se fundamentan en disminuir, reusar, reciclar, disponer y eliminar adecuadamente los RCD, que se traduzca en beneficios para el municipio y su región.

Manejo oportuno de escombros. El primer principio del manejo oportuno de los RCD, recae la responsabilidad inicial de la gestión de los RCD en los diseñadores de las obras civiles, quienes deben seleccionar los materiales de construcción a partir del conocimiento de las consecuencias ambientales, sociales y económicas procurando elegir aquellos que generen menos subproductos en la elaboración, menos residuos en su utilización y sean susceptibles a reusarse o reciclarse. Por cada de RCD que sea sometido al manejo oportuno se ahorra el transporte al sitio de disposición final, se previene la posible disposición inadecuada, se evita la extracción de material natural y se genera empleo. Adicionalmente otro punto crítico en el manejo oportuno de los RCD es el desinterés de los generadores a pagar por la gestión de los residuos productos de la construcción o demolición.

Con el contexto analizado hasta el momento y considerando los aspectos económico, social y ambiental se desarrolla la alternativa manejo oportuno de escombros para la ciudad de Villavicencio que cuenta con las siguientes recomendaciones en cada una de las fases propuestas:

\subsection{En la fuente}

- Uso de materiales alternativos y procesos constructivos que generen menos residuos y/o permitan reciclaje más eficiente.

- Separación de los RC según el potencial de reúso o reciclaje, haciendo uso de contenedores o áreas que impidan la mezcla con otros materiales y/o el ambiente.

- Supervisiones calificadas de la ejecución de la obra y manejo de los RCD generadas.

- Aplicar la normativa ambiental nacional y local vigente para el manejo de los RCD, haciendo énfasis en el decreto 089 del 26 de mayo de 2014, por medio del cual se regula la gestión integral de escombros en el municipio de Villavicencio.

\subsection{En el transporte}

- Renovar el parque automotor.

- Financiar e incorporar a los transportadores informales al pie de fuerza laboral que transporte residuos especiales a centros de acopio temporal.

- Aplicar y controlar la normativa ambiental nacional y local vigente para el manejo de los RCD, haciendo énfasis en el decreto 089 del 26 de mayo de 2014, por medio del cual se regula la gestión integral de escombros en el municipio de Villavicencio. 


\subsection{En el sitio de disposición final}

- Clasificación de los RCD recibidos en acopios temporales para ser direccionados a plantas que se encarguen de su trituración y tamizado.

- El sitio de disposición final deberá contar con una planta de procesamiento de las fracciones de concreto, agregados y mampostería convirtiéndose así no solo en una escombrera sino también en una planta de suministro de agregados provenientes del reciclaje de los escombros.

- Instalación de centros pilotos de acopio temporal de RCD y residuos especiales en diferentes puntos de la ciudad.

\section{Conclusiones}

Estas conclusiones deben enmarcarse por acciones de los diversos actores que promuevan:

- Plan de limpieza de los escombros dispuestos inadecuadamente en la ciudad.

- Programación y aplicación de programas de reducción de RCD en las obras aplicando incentivos financieros.

- Divulgación y puesta en marcha de la normativa vigente.

- Identificación de nuevos sitios que tengan potencial de uso como escombrera para la ciudad.

- Puesta en marcha de una planta de reciclaje de RCD.

- Promoción de un sistema de tarifas para la disposición de RCD en la escombrera autorizada.

- Priorizar el uso de agregados y prefabricados a base de RCD reciclado inicialmente en las obras públicas.

- Campaña de cambio del comportamiento de la ciudadanía dirigido hacia vivir naturalmente.

De esta manera se espera que el escenario local actual en cuanto a los escombros tendrá un cambio de un ciclo lineal de tres fases a uno ciclo circular que permita el manejo oportuno de los RCD generados en la ciudad tal y como se presenta en la figura 4. 
Figura 4

Escenario local versus manejo oportuno

\section{Manejo oportuno de escombros}

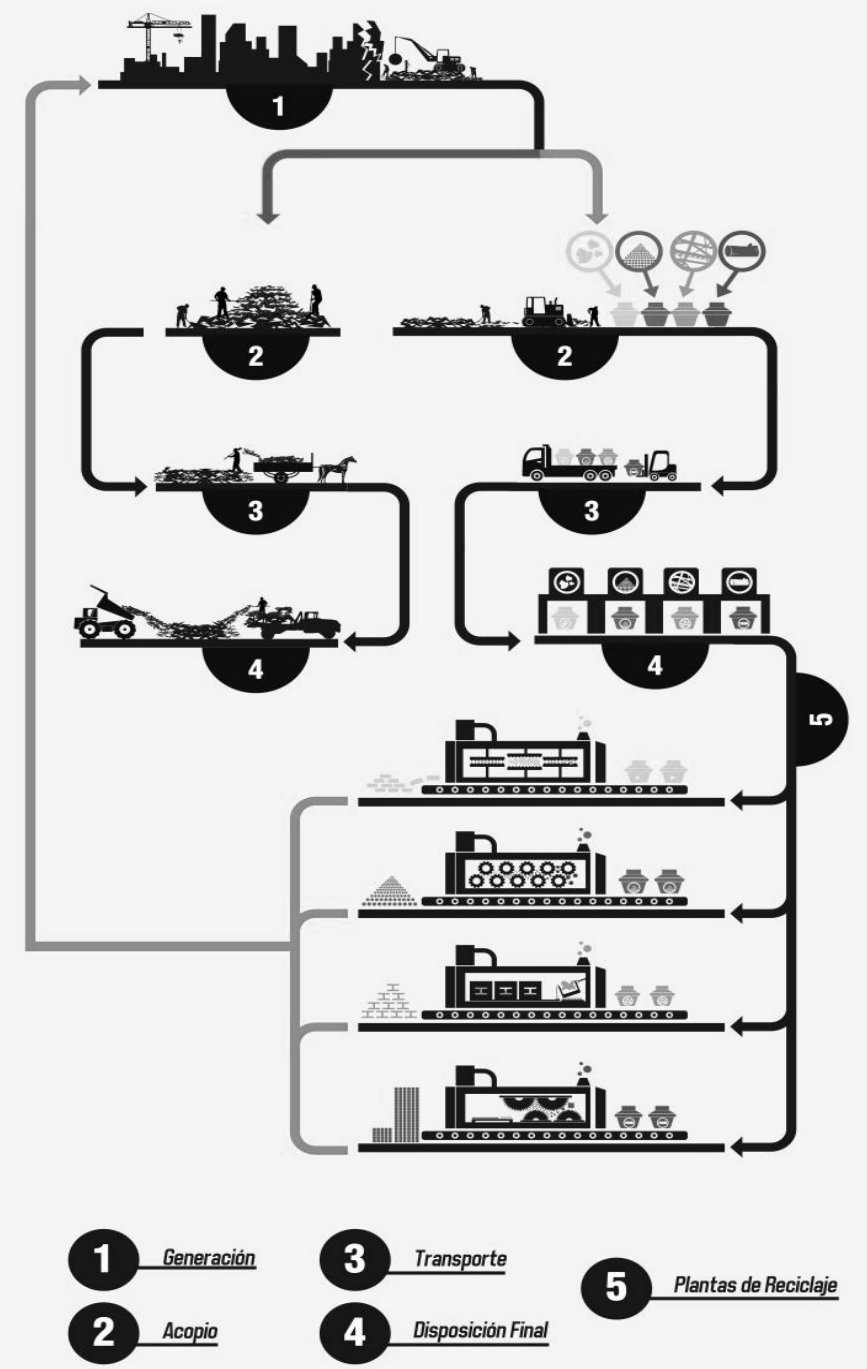

\section{Referencias}

Alencar, L.H., De Miranda Mota, G.M., Alencar, M.H. (2011). The problem of disposing of plaster waste from building sites: problem structuring based on value focus thinking methodology. Waste Manage. 31 (12). P 2512-2521.

Bernardo Miguel, Castilho Gomes Marta, De Brito Jorge. (2016). Demolition waste generation for development of a regional management chain model. Waste Management 49. P 156-169

Castaño, J.O.; Misle Rodríguez, R.; Lasso, L.A., Gómez Cabrera, A.; Ocampo, M.S. (2013). Gestión de residuos de construcción y demolición (RCD) en Bogotá: perspectivas y limitantes. Tecnura. 17. P 121-129.

Chong, W.K., Hermreck, Ch. (2010). Understanding transportation energy and technical metabolism of construction waste recycling. Resour. Conserv. Recycl. 54(9). P 579-590. 
Dahlbo, H.; Bacher, J.; Lähtinen, K.; Jouttijärvi, T.; Suoheimo, P.; Mattila, T.; Sironen, S.; Myllymaa, T.; Saramäki, K. (2015). Construction and demolition waste management a holistic evaluation of environmental performance. J. Cleaner Product. 107. P333-341.

Dane. Departamento Administrativo Nacional de Estadística (2014a). Estadisticas de licenciada de construcción. https://www.dane.gov.co/index.php/estadisticas-por-tema/construccion/licencias-deconstruccion (Consultado en abril de 2014).

Dane. Departamento Administrativo Nacional de Estadística (2014b). Estadisticas de licenciada de construcción. https://www.dane.gov.co/index.php/estadisticas-por-tema/demografia-ypoblacion/proyecciones-de-poblacion. (Consultado en abril de 2014).

De Brito Jorge, Alves Fátima. (2010). Concrete with recycled aggregates: The Portuguese experimental research. Materials and Structures. Vol. 43. P 35-51.

D’appolonia. (1999) Informe preliminar manejo integral de escombros y residuos de construcción. Washington, D.C. Banco Interamericano de Desarrollo. P 127.

Eljaiek Urzola, Mónica; Quiñones Bolaños, Edgar; Mouthon Bello, Javier. (2011). Aprovechamiento de los escombros generados en actividades de demolición de placas de pavimento en Cartagena - Colombia. Hacia la sustentabilidad: Los residuos sólidos como fuente de energía y materia prima. P 558-569.

Fernández Layton S. (2011). Guía metodológica para la reutilización del material de demolición de concreto en obras viales. Disponible desde internet: http://repository.unilibre.edu.co/bitstream/ 10901/5860/1/FernandezLaytonSara2011.pdf. Consultado el 11 de Octubre 2013.

Hoffmann Cathleen, Schubert Sandy, Leemann Andreas, Motavalli Masoud. (2012). Recycled concrete and mixed rubble as aggregates: Influence of variations in composition on the concrete properties and their use as structural material. Construction and building materials. Vol. 35. P 701-709.

Hoornweg Daniel, Bhada-Tata Perinaz. (2012)What a waste a global review of solid waste management. Urban development series Knowledge papers. The world bank.. No. 15. P 16.

Hurtado J. 2000). Metodología de la investigación holística. Caracas. Fundación SYPAL.

MMAE - Ministerio de Medio Ambiente Español. (2001). BOE 166 Plan Nacional de residuos de construcción y demolición 2001-2006, Madrid. 45 Ministerio de Medio Ambiente, 2001. Completar información

Muñoz Alvear Edmundo, FABRES Jara Andrés, CÁRDENAS Ramírez Juan Pablo. (2011). Residuos sólidos del proceso de construcción de viviendas en Chile - cuantificación, caracterización y establecimiento de indicadores. Disponible desde internet: http://www.elecs2013.ufpr.br/wpcontent/uploads/anais/2011/2011_artigo_136.pdf. Consultado el 16 de Noviembre 2013.

Natalini Mario B., Klees Delia R., Tirner Jirina, (2000). Reciclaje y reutilización de materiales residuales de construcción y demolición. En: http://www.unne.edu.ar/unnevieja/Web/cyt/cyt/2000/7_ tecnologicas/t_pdf/t_013.pdf. Completar información

Pacheco-Torgal, F., Tam, W.M.Y., Labrincha, J.A., Ding, Y., De Brito, J. (2013). Handbook of recycled concrete and demolition waste. Woodhead Publishing. P 672.

Rodríguez G., Medina C., Alegre F.J., Asensio E., Sánchez De Rojas M. I. (2015). Assessment of construction and demolition waste plant management in Spain: in pursuit of sustainability and eco-efficiency. Journal of Cleaner Production. Vol. 90. p 16-24. 
Serrano, M.F, Pérez D. (2009). Propuesta de un programa de gestión integral de escombros. II Simposio I Iberoamericano de Ingeniería de Residuos. Universidad del Norte Barranquilla.

Silva Yimmy, Robayo Rafael, Mattey Pedro, Delvasto Silvio. (2015). Obtención de concretos autocompactantes empleando residuos de demolición. Revista LatinAm. Metal. Mat. Vol 35. P 86-94.

Sui Yuwu, Mueller Anette. (2012). Development of thermo-mechanical treatment for recycling of used concrete. Materials and Structures. Vol. 45. P 1487-1495.

Triola, Mario F. (2009). Estadística. Pearson education. Décima edición. México. páginas?

Vásquez Hernández Alejandro, Botero Botero Luis Fernando, Carvajal Arango David. (2015) Fabricación de bloques de tierra comprimida con adición de residuos de construcción y demolición como reemplazo del agregado pétreo convencional. Ingeniería y Ciencia. Vol. 11 No. 21. P 197-220.

Vergel G. (2010). Metodología, un manual para la elaboración de diseños y proyectos de investigación. Compilación y ampliación temática. Barranquilla. Publicaciones Corporación UNICOSTA.

Wrap. (2012). Working togethers for a world without waste, Construction Applications. http://aggregain.wrap.org.uk/ opportunities/applications (consultado en abril de 2014)

Yeheyis, M., Hewage, K., Alam, M.S., Eskicioglu, C., Sadiq, R. (2013). An overview of construction and demolition waste management in Canada: a lifecycle analysis approach to sustainability. Clean Technol. Environ. Policy. Vol. 15 (1). P 81- 91.

Costafreda Mustelier Jorge Luis, Calvo Pérez Benjamín, Peralta Orellana Xiomara Beatriz. (2013). Aprovechamiento de los residuos de demolición de la ciudad de Madrid en morteros con zeolita natural: una contribución a la mejora del medio ambiente. http://oa.upm.es/1414/1/COSTAFREDA_PON_2009_01.pdf

Hoffmann Cathleen, Schubert Sandy, Leemann Andreas, Motavalli Masoud. (2012). Recycled concrete and mixed rubble as aggregates: Influence of variations in composition on the concrete properties and their use as structural material. Construction and Building Materials. Vol. 35. P 701-709.

Esta obra está bajo una Licencia Creative Commons Attribución-NoCommercial 4.0 International

\section{$(\mathrm{cc}) \mathbf{B Y}$-NC}

